\title{
ANÁLISIS DE LAS CAUSALES EXPLICATIVAS EN UN CORPUS ORAL ${ }^{1}$
}

\author{
Otilia PÉREz GIL \\ Grupo de investigación «Sociolingüistica, Dialectología \\ y Lingüistica aplicada a la enseñanza de la lengua» \\ Universidad de Las Palmas de Gran Canaria (España)
}

\section{RESUMEN}

En este trabajo analizamos las construcciones causales registradas en un corpus oral de Las Palmas de Gran Canaria, elaborado en el marco del PRESEEA (Proyecto de estudio sociolingüístico del español de España y de América). Para ello realizamos, en primer lugar, una propuesta de clasificación de esos enunciados, insistiendo especialmente en la necesidad de diferenciar los conceptos de 'causa de la enunciación' y 'causa explicativa'. A continuación, profundizamos en el estudio de las que constituyen el grupo más numeroso: las causales explicativas. Con este fin, atendemos a los contextos que propician su aparición, junto a otros factores que pueden incidir en su elevada frecuencia de uso.

PALABRAS CLAVE: enunciados causales, causa explicativa, oralidad, PRESEEA, español de Canarias.

\section{AbSTRACT}

In this study, we shall analyse causal constructions in the light of an oral corpus from Las Palmas de Gran Canaria which was compiled within the framework of the PRESEEA research group ('Project for the Sociolinguistic Study of Spanish from Spain and America'). To this end, we shall offer, in the first place, a proposal for the classification of this type of utterance, with special emphasis on the need to differentiate the concepts of 'utterance cause' and 'explicative cause'. Next, we shall concentrate on a fine-grained analysis of the most frequent type, namely, explicative causal utterances. With this objective in mind, we shall focus

${ }^{1}$ Esta publicación forma parte del proyecto de investigación «Estudio complementario de los patrones sociolingüísticos del español de España-Las Palmas de Gran Canaria (ECOPASOS)» (Referencia FFI2015-68171-C5-5-P), financiado por el Ministerio de Economía y Competitividad de España y por el Fondo Europeo de Desarrollo Regional (FEDER). 
on the context which favour their use, together with other factors which might influence their high frequency of use.

KEYWORDS: causal utterances, explicative cause, oral production, PRESEEA, Spanish spoken in the Canary Islands.

\section{INTRODUCCIÓN}

Desde las primeras observaciones de Bello ([1847] 1981: 578) sobre la necesidad de diferenciar dos tipos de porque, uno adverbial y otro conjuntivo (este último, introductor de una «causa lógica»), las reflexiones en torno a la existencia de diversas clases de causales han sido una constante en los estudios gramaticales sobre estas oraciones. Tras una primera etapa en la que, con muy pocas excepciones ${ }^{2}$, se adoptó para el español la misma división de las gramáticas latinas (causales «coordinadas»y «subordinadas»), la publicación, en 1978, del conocido trabajo de Lapesa sobre «dos tipos de subordinación causal» supuso un punto de inflexión en el tratamiento de esta cuestión. El recurso a la oposición enunciado/enunciación, fundamento de su distinción entre las causales I y II, constituye hoy una premisa en la mayoría de los estudios sobre estas construcciones, que se ha visto respaldada por su inclusión, por vez primera, en la gramática académica (RAEASALE 2009).

Pero, junto a las causales del enunciado y de la enunciación, la NGLE da entrada, igualmente, a otro conjunto causal al que dedica un apartado específico: las «causales explicativas». Este otro subgrupo de causales, sobre las que la Academia advierte que «no constituyen un tipo marginal de oraciones causales en español ni en otras lenguas», ha sido estudiado en profundidad por Santos Río (1981) y Gutiérrez Ordóñez (2000), quienes, desde distintas ópticas, han demostrado el carácter imprescindible del rasgo 'explicativo' en el análisis de la expresión de la causa.

En un trabajo precedente donde nos ocupamos del estudio de los enunciados con porque en la oralidad (Pérez Gil 2017) pudimos comprobar cómo, efectivamente, las explicativas, lejos de ser un grupo minoritario, constituyen el tipo de causales con mayor frecuencia de uso en el habla ${ }^{3}$. En la presente investigación, en la que sumamos, a los datos de porque, los enun-

2 Cfr. Gili Gaya (1943), Sánchez Márquez (1972) y, como consecuencia de la participación del primero en la redacción del Esbozo, RAE (1973).

${ }^{3}$ En concreto, el $83,2 \%$ de los casos allí registrados pertenecían a causales explicativas. 
ciados introducidos mediante otras fórmulas causales, nos proponemos precisar la importancia cuantitativa de esta clase de causales e indagar en los factores que favorecen su predominio en el discurso. Previamente, exponemos los criterios aplicados para clasificar los enunciados causales de nuestro corpus, donde desempeña un papel fundamental la relación existente entre las causales explicativas y la oposición enunciado/enunciación.

\section{PRecisiones teóricas PREVIAS}

\subsection{Causales de la enunciación y causales explicativas}

Como es sabido, la clasificación formulada por Lapesa (1978), que hunde sus raíces en la obra de Bello ${ }^{4}$, consiste en diferenciar las causales referidas a lo dicho («El suelo está mojado porque ha llovido») de aquellas otras portadoras de la razón que se ha tenido para decirlo ( Ha llovido, porque el suelo está mojado»). Estas últimas, que se reformularían, para su análisis, recuperando un verbo implícito («[Digo que] ha llovido porque el suelo está mojado»), han recibido después la denominación de "causales de la enunciación» ${ }^{5}$, en oposición a las primeras, «causales del enunciado». En cambio, quienes contemplan en sus estudios las causales explicativas ${ }^{6}$ fundamentan su reconocimiento en un matiz semántico menos restrictivo que la 'causa de la enunciación', al dar cabida en ellas a toda causal aducida a modo de explicación o justificación ${ }^{7}$.

Pese a la diferente base en la que se sustentan ambas clasificaciones, ha sido muy recurrente, en la bibliografía, la equiparación de las causales explicativas con las de la enunciación. En unas ocasiones, la confusión viene dada al interpretar la oposición explicativa/no explicativa definida por

${ }^{4}$ De hecho, parte del significativo cambio que el gramático venezolano realizó en la revisión de su Gramática al incluir, entre las características propias del porque conjuntivo, su capacidad para expresar «el fundamento que hemos tenido para enunciar» la proposición no causal ([1847] 1981: 578).

5 También se les han atribuido otros nombres, como los de «causales lógicas», cuya inadecuación es advertida en la NGLE (RAE-ASALE 2009: 3472-3473), «causales de dicto» (Santos Río 1993) o "causales de verbo enunciativo», etiqueta, esta última, preferida por Iglesias Bango (1997) y Gutiérrez Ordóñez (2000).

${ }^{6}$ Si bien son los ya citados Santos Río y Gutiérrez Ordóñez quienes otorgan una entidad diferenciada a las causales explicativas, las referencias al carácter explicativo de determinadas causales están ya presentes, por ejemplo, en los trabajos de S. García (1996) y Galán (1995 y 1999).

7 La NGLE ejemplifica este concepto con un enunciado, entre otros, como «Todas las luces estaban apagadas, pues se esperaba un ataque de la aviación» (RAE-ASALE 2009: 3475), donde puede advertirse claramente que esa justificación no necesariamente ha de referirse a la enunciación. 
Santos Río (1981) como similar, en el plano conceptual, a la establecida anteriormente por Lapesa $(1978)^{8}$; en otras, el uso sinonímico de ambas expresiones se amplía a otros términos surgidos en distintos marcos teóricos:

... las oraciones de la enunciación, indirectas, explicativas, periféricas, o como se las quiera llamar, están marcadas por una pausa obligatoria... (Caravedo 2003: 47).

Incluso la Academia, que hoy afirma que «no parece que estas dos nociones sean enteramente equivalentes» (RAE-ASALE 2009: 3475), llegó a asimilar, antes de la publicación de la NGLE, esos dos tipos de causales: «Se escribe coma [...] ante causales lógicas o explicativas, también llamadas "de la enunciación”»(DPD s. v. coma).

La imprecisión que conlleva interpretar que las causales explicativas tienen una correspondencia exacta con las causales de la enunciación ha sido puesta de manifiesto tanto por Gutiérrez Ordóñez, quien argumenta la «defensa de las causales explicativas como un grupo diferente de las causales de verbo enunciativo» (2000: 67) ${ }^{9}$, como por Santos Río, que ha rechazado explícitamente la identificación de su propuesta con la de Lapesa ${ }^{10}$. En ese sentido, cabe subrayar cómo Santos Río (1981) incluye, entre sus causales explicativas, ejemplos del tipo «Como soy pequeño, no tengo problemas con los techos» y «No se veían bien las pisadas, pues era de noche» -según Lapesa, causales tipo II o del enunciado-. Pero entre sus causales explicativas contempla, también, casos como «Ya que tenemos el día libre, vámonos de campo» $\mathrm{y}$ "Quédate, que ahora viene lo mejor». Si los dos primeros ejemplos revelaban la diferente naturaleza de las causales «explicativas» y «de la enunciación», estos otros -en los que la causa apunta a la enunciación- permiten deducir que, aun tratándose de conceptos diferentes, no dejan de ser compatibles ${ }^{11}$. Esta observación tiene refrendo en la NGLE, donde se advierte que «las causales explicativas están relacionadas con las causales de la enunciación, pero no son por completo equivalentes a ellas» (2009: 3474).

Un enfoque diferente presenta Gutiérrez Ordóñez (2000), para quien las causales explicativas constituyen un grupo independiente de las «de

8 Vid., por ejemplo, Narbona (1990) o López (1994).

${ }^{9}$ La misma intención ha dejado reflejada en otros trabajos (vid., por ejemplo, Gutiérrez Ordóñez 2002).

10 «En un trabajo de hace ya bastantes años describí una distinción entre causales explicativas y causales propiamente dichas [...]. Esta distinción [...] se me ha atribuido a veces de forma totalmente incorrecta, convirtiéndola en equivalente a la famosa distinción recordada por Rafael Lapesa» (Santos Río 1993: 33).

${ }^{11}$ La confluencia de la causa 'explicativa' y 'de la enunciación' en esos enunciados es corroborada por Santos Río en un trabajo posterior (vid. Santos Río 2001: 457). 
verbo de enunciado» y "de verbo enunciativo» ${ }^{12}$. Su noción de "causal de verbo enunciativo', no obstante, abarca únicamente las causales «lógicas» o «hipotéticas» («Pepe fuma, porque tiene el cenicero lleno de colillas»), que expresan el «fundamento en el que basamos la hipótesis que formulamos en la oración principal» (2000: 114). En este aspecto también se distancia, por tanto, de Santos Río, pues ejemplos como «Ya que tenemos el día libre, vámonos de campo" y "Quédate, que ahora viene lo mejor» serán considerados por Gutiérrez Ordóñez explicativos, pero no causales de dicto. Esas diferencias a la hora de delimitar el grupo de las causales explicativas, así como su relación con las causales del enunciado y de la enunciación, pueden haber contribuido a la errónea identificación de causales explicativas y de la enunciación. A ello se añaden otros factores como su indudable cercanía semántica -en cuanto que las causales de la enunciación no dejan de aportar una justificación o explicación ${ }^{13}-$, su supuesta similitud formal (ambas son consideradas por la RAE-ASALE, en su NGLE, causales «externas al predicado») y un falso paralelismo surgido a raíz de la pertenencia de todas las causales no explicativas al grupo II de Lapesa $(1978)^{14}$.

\subsection{Propuesta de clasificación de los enunciados causales}

En consecuencia con las consideraciones expuestas anteriormente, el modelo de clasificación que aplicamos a nuestro corpus tiene en cuenta la diferencia existente entre los conceptos de 'causa explicativa' y 'causa de la enunciación', por lo que resulta de la combinación de la oposición enunciado/enunciación ${ }^{15}$ y la presencia o no del rasgo 'explicativo'. Con estas premisas, diferenciamos los siguientes grupos:

${ }^{12}$ Admite, sin embargo, que con estas últimas «en ocasiones las diferencias no son muy evidentes» (Gutiérrez Ordóñez 2000: 111). Prueba de ello es que, al tratar las explicativas referidas a un segmento discursivo, considere necesaria la recuperación del verbo locutivo decir. «EN MI CASA, [digo en mi casa] porque (pues, puesto que) esta no es la mía, yo actuaría con mayor libertad» (2000: 136); o su afirmación posterior de que dichas causales pueden introducir una justificación «de re o de dicto» (2000: 149).

${ }^{13}$ En referencia a dichas causales escribió Lapesa que «el hecho innegable de que en estas secuencias las suboraciones causales justifiquen o expliquen el acto enunciativo del hablante hizo pensar que denotaban la "causa lógica”..." (1978: 204; la cursiva es nuestra).

${ }^{14}$ De acuerdo con Gutiérrez Ordóñez, la coincidencia -en ese sentido- de las no explicativas de Santos Río (1981) con las causales del enunciado descritas por Lapesa (1978) «hizo que muchos autores efectuaran una asimilación en la parcela complementaria: causales de causa lógica $=$ causales de la enunciación = causales explicativas = causales periféricas» (2000: 109).

${ }^{15}$ Aunque esa separación bipartita se ha mostrado insuficiente para clasificar los enunciados causales en la oralidad (vid., por ejemplo, Carbonero 2001 y Caravedo 2003), resulta necesaria, desde nuestro punto de vista, para realizar una descripción lo más completa posible de dichos enunciados. 
1. Causales del enunciado

1.1. No explicativas

1.2. Explicativas

2. Causales de la enunciación (explicativas)

Como puede apreciarse en el esquema, seguimos el criterio de Santos Río (2001) al entender que las dicotomías enunciado/enunciación y no explicativa/explicativa no son excluyentes entre sí, pues el valor explicativo de una causal puede ir orientado tanto al enunciado como a la enunciación. Consideramos, asimismo, que las causales de la enunciación conforman un tipo específico de las causales explicativas -aquel en el que lo justificado por el hablante es su propio acto verbal-. Así como no cabe la identificación de esos dos conceptos, ya que no todas las explicativas son causales de la enunciación, tampoco es posible separarlas en dos grupos completamente independientes, puesto que en las causales de la enunciación subyace siempre la intención explicativa ${ }^{16}$. Se trata, por lo demás, de una propuesta que tiene cabida en la actual teoría gramatical de la RAE-ASALE: «Unos autores separan las causales de la enunciación de las causales explicativas, pero otros consideran las primeras como una mera variante de las segundas» (2009: 3476) ${ }^{17}$.

Cabe advertir que, entre las causales de la enunciación, incluimos las referidas a un fragmento discursivo no asertivo, como son las causales «directivas» del tipo «Callaos, que molestáis a papá» o «Ven, que ya he resuelto el problema» -para Gutiérrez Ordóñez, catalogables en las causales explicativas y diferentes a las causales de verbo enunciativo (2000: 123)-. Su vinculación con las causales de la enunciación fue ya aceptada por Lapesa al contemplar en sus causales del grupo I (correspondiente a las de la enunciación) ejemplos como «No me esperes, que hoy llevo otro camino» o «No te rías, que no tengo ganas de bromas» (1978: 183), y es señalada hoy en día por la RAE-ASALE: «Se ha observado que, en general, las causales explicativas con porque que se usan en contextos no asertivos se asimilan a las causales de la enunciación: ¡Sal pronto!, porque ya suena la campana» (2009: 3464).

Aunque proporcionaremos los datos cuantitativos referidos a todos los grupos delimitados, nos centraremos en el análisis de las causales contenidas en 1.2 (explicativas del enunciado) y 2 (explicativas de la enunciación).

${ }^{16}$ De ahí las dificultades surgidas cuando se ha pretendido establecer una separación estricta entre causales explicativas y de la enunciación (vid. Grande Alija y Lanero Rodríguez 2019).

${ }_{17}$ Más adelante, al tratar las causales introducidas por que («Ojalá termine pronto, que ya me estoy cansando»; «Enhorabuena, que me he enterado de que conseguiste el puesto»), la Academia afirma que «estos usos podrían interpretarse como argumento a favor de que las causales de la enunciación constituyen un grupo particular de las causales explicativas» (2009: 3478). 


\section{Aspectos metodolóGicos}

Para llevar a cabo nuestro estudio, nos basamos en los enunciados causales extraídos de un corpus oral correspondiente a doce grabaciones realizadas a otros tantos hablantes de Las Palmas de Gran Canaria. Las muestras lingüísticas analizadas, que en conjunto suman un total de 11 horas, han sido recogidas en el marco del «Proyecto para el estudio sociolingüístico del español de España y de América» (PRESEEA), coordinado por Francisco Moreno. Las entrevistas, por tanto, que fueron realizadas por el equipo de investigación de la Universidad de Las Palmas de Gran Canaria, responden a las pautas establecidas en dicho proyecto, por lo que son semidirigidas y se grabaron con el consentimiento previo de los informantes (vid. Hernández Cabrera y Samper 2012). Todos los sujetos de la muestra se sitúan en el nivel más elevado de formación definido en las bases metodológicas del PRESEEA (enseñanza superior) ${ }^{18}$ y presentan una distribución equitativa en cuanto a la edad y el sexo, tal como se refleja en el siguiente cuadro:

CuAdro 1. Distribución de los informantes ${ }^{19}$

\begin{tabular}{cccc}
\hline Generaciones & Hombres & Mujeres & $\mathrm{N}$ \\
\hline $1 .^{\mathrm{a}}(20-34$ años $)$ & 2 & 2 & 4 \\
\hline $2 .^{\mathrm{a}}(35-54$ años $)$ & 2 & 2 & 4 \\
\hline $3 .^{\mathrm{a}}(55$ años o más $)$ & 2 & 2 & 4 \\
\hline $\mathrm{N}$ & 6 & 6 & 12 \\
\hline
\end{tabular}

$\mathrm{Al}$ fundamentar nuestras observaciones en los enunciados obtenidos de un corpus oral, nos alejamos del proceder habitual en los estudios gramaticales, donde los presupuestos teóricos expuestos suelen sustentarse en ejemplos que, ya sean tomados de textos de diversa índole (literaria, periodística, etc.), ya provengan de la introspección lingüística del autor, han sido formulados en virtud de las normas del código escrito. Este modus operandi-que continúa presente, por ejemplo, en la $N G L E-$ ha provocado a

${ }^{18}$ Se trata de informantes con estudios universitarios y un mínimo de quince años de escolarización.

${ }^{19}$ Para su identificación nos serviremos de una sucesión de códigos referentes a su lugar de origen (LP), sexo (H/M) y grupo etario al que pertenecen (1/2/3), a los que finalmente añadiremos una letra minúscula $(\mathrm{a} / \mathrm{b})$ para diferenciar entre los dos informantes ubicados en la misma casilla (es decir, con idénticas características en cuanto a las variables extralingüísticas contempladas). 
menudo desajustes entre los rasgos tradicionalmente atribuidos a determinadas causales en la teoría gramatical y los que aquellas presentan en el habla ${ }^{20}$. Un análisis empírico basado en casos reales, además de evitar la subjetividad implícita en la selección -e incluso invención- de ejemplos ad hoc, nos permitirá conocer las diversas posibilidades expresivas que ofrecen las causales explicativas -del enunciado y de la enunciación- en español y determinar con mayor precisión sus principales contextos de uso, así como abordar la función que esta clase de causales cumple en el discurso.

\section{Datos cuantitativos}

El proceso de identificación de los enunciados causales en las muestras estudiadas $^{21}$ nos ha permitido extraer para nuestro análisis un total de 885 casos, que presentan la distribución recogida en el cuadro 2.

CuAdro 2. Clasificación de los enunciados causales

\begin{tabular}{lcc}
\hline \multicolumn{1}{c}{ TIPOS DE CAUSALES DIFERENCIADOS } & N & $\%$ \\
\hline Causales del enunciado no explicativas & 129 & $14,6 \%$ \\
\hline Causales del enunciado explicativas & 541 & $61,1 \%$ \\
\hline Causales de la enunciación (explicativas) & 215 & $24,3 \%$ \\
\hline Total & 885 & \\
\hline
\end{tabular}

El grupo mayoritario es, con diferencia, el formado por las causales del enunciado explicativas, al que pertenecen más de la mitad de los enunciados analizados (un 61,1\%). Le siguen, atendiendo a su frecuencia de uso, las causales de la enunciación, que representan casi la cuarta parte del conjunto (un 24,3\%), mientras que las no explicativas suman, únicamente, el $14,6 \%$ del total. Las cifras resultantes, por tanto, corroboran de forma

${ }^{20}$ Es este el caso, por ejemplo, de la pausa, constantemente vinculada a las causales de la enunciación y a las explicativas, en contraste con las observaciones realizadas por los estudiosos de la oralidad, que han constatado la falta de sistematicidad de ese recurso prosódico en los contextos causales (cf. Carbonero 2001 y Caravedo 2003). Se trata de un tema del que nos hemos ocupado de forma pormenorizada con anterioridad, realizando un estudio acústico de los índices suprasegmentales influyentes en el reconocimiento de cada tipo de causal (vid. Pérez Gil 2017).

${ }^{21}$ Para un conocimiento detallado de los criterios aplicados en la localización y posterior cuantificación de los casos objeto de estudio, vid. Pérez Gil (2017: 181-189). 
innegable el predominio, en el habla, de los enunciados causales explicativos, ya sean del enunciado -como sucede en más del $70 \%$ de los casoso de la enunciación:

GRÁFICA 1. Causales explicativas y no explicativas
GrÁFICA 2. Causales explicativas del enunciado y de la enunciación

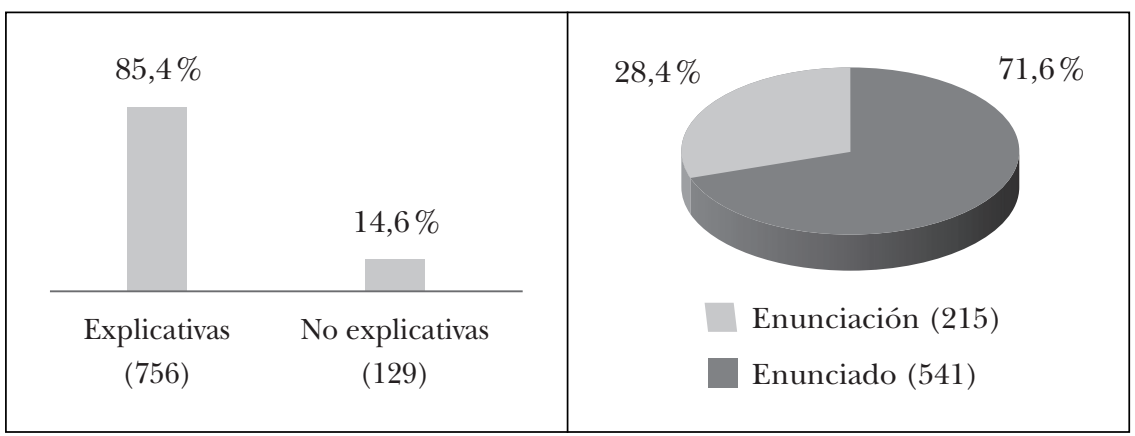

\section{ANÁLISIS DE CONTEXTOS Y OTROS POSIBLES FACTORES CONDICIONANTES}

El elevado porcentaje de uso alcanzado por las causales explicativas en nuestro corpus, según el cual en el $85,4 \%$ de las ocasiones en las que un hablante recurre a una construcción causal lo hace con la intención de proporcionar algún tipo de explicación o justificación, pone de manifiesto la importancia que adquieren estas construcciones en el desarrollo de la conversación. El análisis cualitativo de todas las causales explicativas extraídas de nuestro corpus nos ha permitido reconocer cuáles son los contextos habituales en los que estas aparecen y definir, para su sistematización, las categorías que a continuación proponemos ${ }^{22}$ :

1) Expresión de opiniones o juicios personales, que a menudo giran en torno a un tema polémico, susceptible de generar algún conflicto con el interlocutor:

(1) ... que haya salido una ley antitabaco (1") me resul-/ me parece a mí que es bueno por/ por dos motivos// es bueno/ para el resto de los no fumadores// de las personas no fumadoras/ y es/ bueno/ para el mismo fumador/ porque puede ser que le motive// a dejar de fumar (LPM2a)

${ }^{22}$ Es esta, no obstante, una primera propuesta que, en el futuro, podrá ser matizada o ampliada con los datos que aporte el estudio de otros corpus. 
(2) ... lo que me parece:// que fastidia las cosas es la inmigración ilegal// porque te: te crea una situación de economía sumergida (1”) (LPH1a)

En otras ocasiones, el hablante trata de justificar gustos y preferencias personales, como en (3), o la valoración de algún hecho, como en (4):

(3) I: [...] Nabokov que me gusta mucho y:// o el de toda la vida incluso el Chéjov este [que de: de:]

E: [Chéjov]

I: de estas cosas así// fundamentalmente porque a mí siempre me han gustado/cuando/ ahora se han puesto de moda pero a mí siempre me gustaron/ de toda la vida// me gustaron los cuentos cortos (LPH2b)

(4) ... me pareció el viaje interesantísimo// porque fue un viaje que sin ir en crucero// viajamos siempre en barco// (LPM3a)

2) Toma de decisiones:

(5) ... como me habian// preavisado un poco de que el nivel/ general era bajo pues// dije "no no// las matemáticas y tal"/ pedí las más fuertes (LPH1a)

Dichas decisiones pueden referirse al propio discurso, como en (6), donde se justifica la elección del tema que se va a tratar, o incluso a una situación hipotética, como la formulada por el informante en (7) al habérsele preguntado a qué dedicaría su tiempo si ganara un premio de lotería:

(6) ... te describo mi: la casa de mis padres aquí en Las Palmas// porque digamos es en donde: he estado más años// (LPH1b)

(7) ... me dedicaría a escribir descaradamente y a perfec- a perfeccionar no solo (1") mi nivel técnico/ o sea mi nivel de: de capacidad de:// de/ de filología ¿no? de saber/ de lengua de idioma y todo esto// porque me quedé s- eso creo que tengo un déficit importante/ (LPH2b)

3) Respuestas negativas (o afirmativas, en su caso) a preguntas directas del interlocutor:

(8) E: [...] aunque tú sacaste un buen número no obtuviste plaza en Canarias ¿̇o sí?

I: no:/ no obtuve en Canarias/ porque no salía ninguna en Canarias// (LPM3a)

4) Instrucciones o recomendaciones:

(9) ... entonces se fríen primero// porque si se guisan directamente se pueden desmoronar// (LPM2b) 
5) Observaciones realizadas a modo de advertencia (en el ejemplo, reforzada con la partícula fática $\dot{i} e h$ ?):

(10) ... a él no le gustaba mucho ver niños/ ¿eh?// porque la patología de los niños es como má:s complicada (LPM3b)

6) Informaciones que el informante considera conveniente acompanar de algún comentario aclaratorio o explicativo:

(11) I: [...] él/ al padecer de alergia al polvo y a los// a los (m:)

$\mathrm{E}$ : ácaros

I: ácaros ácaros// pues efectivamente él no puede tener demasiado libro en la habitación/ (LPH3a)

Esa justificación se siente especialmente necesaria cuando, ante una petición de información, el hablante no dispone de los datos necesarios para cumplir las expectativas de su interlocutor:

(12) E: [...] ¿hay/ hay más cafeterías ahora o más bares que antes? o: o co- ¿qué/ qué piensas? / ¿no sabes?/

I: no sé ${ }^{23}$ porque yo ahora el/ (m:) plan cafeterías como que no he ido mucho ahora/ ¿entiendes?// (LPM3b)

Asimismo, las causales explicativas suelen utilizarse como un recurso con el que salvaguardar la propia imagen cuando la información dada podría cuestionarla o perjudicarla en algún aspecto (en el primero de los dos casos que ofrecemos, la informante trata de justificar la actitud de distanciamiento que adopta en el desempeño de su labor profesional; en el segundo, el desfase en sus conocimientos sobre la materia de la que impartía docencia):

(13) E: y y tú las clases ¿cómo las das ahora? o sea/ (e:)/ co- no como cuando estabas [en Tafira]

I: $[$ no]/ no/

E: o sea

I: me implico: bastante menos// abrevio bastante más// (m:) porque:// cada vez los resultados son peores// (LPM2b)

(14) E: porque: claro tenías que como que [actualizarte:]

I: [sí me tenía] necesité actualizarme ${ }^{24}$ es que los años del bachillerato a distancia fueron un bajón en mis conocimientos [horrible] (LPM3a)

La aparición de una causal explicativa es frecuente, igualmente, si la información que se transmite se caracteriza por su alto grado de especialización en una profesión o rama de conocimiento:

\footnotetext{
${ }^{23}$ Aunque no hay pausa, sí se produce una inflexión tonal descendente antes de porque.

24 Debe considerarse la existencia, en este punto, de una frontera entonativa.
} 
(15) ... lo que son las viguetas pues siempre/ se mantienen// igual que los encofrados de la:s vigas// se mantienen con puntales metálicos// porque// no son autoportantes ¿no? sino que hay que// digamos apuntalar e:l/ el forjado// (LPH2a)

Del mismo modo, es habitual que el informante juzgue conveniente, para la mejor comprensión de una información, dar a conocer un nuevo dato por medio de la causal explicativa:

(16) ... recuerdo especialmente:// (e:) paseos con mis padres por el muelle// para ir a ver los barcos// que a mi padre le encantan los barcos// (LPM2a)

Por último, en el apartado de las informaciones no es extraño encontrar ejemplos en los que la explicación constituye un comentario de índole lingüística, que normalmente aclara el origen o significado de un término (17), aunque puede también servir de fundamento a una consideración previa sobre la idoneidad de una expresión (18):

(17) I: [... continúas al fondo hay una puerta de madera// y entras al/ nosotros lo llamamos el patio/

$\mathrm{E}:[(\mathrm{hm}:)]$

I: [porque] antes era un patio abierto/ y ahora está techado// (LPM2a)

(18) ... a pesar de que su familia// la familia de don X// siempre fue (1") no di- no quiero decir anticlerical/ porque n:o era anticlerical era muy respetuosa con las ideas de los demás// pero sí era: una familia totalmente atea (LPH3a)

7) Conclusiones y reflexiones derivadas de algún hecho expresado en la causal explicativa:

(19) ... ahora pienso "los pobres profesores que hacían lo que podían"// si llegamos a llegábamos a ser yo creo que hasta cerca de sesenta// hasta cerca de sesenta en cada aula/ (LPH2b)

8) Comportamiento o actitud mostrada ante una circunstancia:

(20) ... ya tienen el café hecho y:// y sobre todo mi compañera: X pues lo suele agradecer mucho// porque dice que nadie le hace mimos// (LPH1a)

9) Explicativas con como suspendidas, en las que lo justificado se da por sobreentendido (vid. Montolío 2001):

(21) lo he le- leído en la prensa no tengo seguridad porque como ya no me interesa// (LPM3a)

A la relación anterior debe sumarse, aún, un último grupo: el de las causales de la enunciación (que, como ya hemos argumentado, son expli- 
cativas donde lo justificado es el propio acto enunciativo). La distribución porcentual de todos ellos nos ofrece los siguientes resultados:

\section{CuAdro 3. Contextos de aparición de las causales explicativas}

\begin{tabular}{|lrr|}
\hline REFERENTE DE LA JUSTIFICACIÓN (756 casos) & N & $\%$ \\
\hline 1. Opiniones y juicios personales & 85 & 11,3 \\
\hline 2. Toma de decisiones & 43 & 5,7 \\
\hline 3. Respuestas negativas o afirmativas & 19 & 2,5 \\
\hline 4. Instrucciones o recomendaciones & 1 & 0,1 \\
\hline 5. Observaciones a modo de advertencia & 11 & 1,5 \\
\hline 6. Informaciones & 288 & 38,1 \\
\hline 7. Conclusiones y reflexiones & 53 & 7 \\
\hline 8. Comportamientos o actitudes & 32 & 4,2 \\
\hline 9. Omitido por sobreentenderse & 9 & 1,2 \\
\hline 10. Acto enunciativo (causales de la enunciación) & 215 & 28,4 \\
\hline
\end{tabular}

Los datos, por tanto, nos indican que es la transmisión de una información (con un porcentaje del 38,1\%) el contexto que más favorece el empleo de una causal explicativa ${ }^{25}$, seguido de las explicaciones alusivas a la enunciación (que, como ya quedó recogido en la gráfica 2, suponen el $28,4 \%$ de todas las causales explicativas). De hecho, ambos contextos aúnan el 66,5\% del total de casos registrados, mientras que, de los restantes factores contextuales diferenciados, solo uno -el de las explicativas ligadas a un juicio u opinión personal- presenta una incidencia superior al $10 \%$.

Ante el interés que suscitan las causales de la enunciación en las descripciones gramaticales y su significativa presencia en las muestras estudiadas, nos parece especialmente relevante atender a las diversas posibilidades que presentan en la oralidad. A partir de la información que nos proporciona nuestro corpus, se deduce que la explicación justificativa de un acto de habla se ajusta, normalmente, a una de las siguientes opciones:

${ }^{25}$ Las informaciones forman parte de la relación de actos de habla que Gutiérrez Ordóñez (2000) vincula al uso de las explicativas, en la que también encontramos otras posibilidades que corrobora el análisis de nuestro corpus, como son las justificaciones de decisiones o de determinadas respuestas. 
1) Causales lógico-deductivas. También llamadas «hipotéticas» (Galán 1995 y 1999), responden al prototipo que más se repite en las gramáticas para ilustrar las causales de la enunciación («Ha llovido, porque el suelo está mojado») y coinciden, asimismo, con las «causales de verbo enunciativo» definidas por Gutiérrez Ordóñez (2000):

(22) ... el olor// (m:) no debía de ser (m:) desagradable ni fuerte// porque: (e:) no lo recuerdo (LPH3a)

2) Prueba o constatación. La causal no se toma ya como base para la formulación de una hipótesis -como sucede en el grupo anterior-, sino que es esgrimida, a modo de prueba, con el fin de reforzar el fundamento de la enunciación a ojos del interlocutor:

(23) ... I: [...] los patios estaban separados// primero estaban las niñas/ y luego estaban los niños//

$\mathrm{E}:$ (hm:) (hm:)//

I: y: en-/ tenían entradas separadas/ y cuando nos veíamos era en la salida/

E: sa- ajá//

I: y: ni/ ni siquiera te dejaban que te vieras en el recreo ${ }^{26}$ porque://

E: [claro]

I: [si] veían que te acercabas a la zona te iban corriendo [a llamar la atención] (LPM3b)

3) Paráfrasis. Mediante la causal se ejemplifica o reitera un contenido similar al del segmento discursivo cuya enunciación se pretende justificar:

(24) ... lo han hecho en un barranco/ de:// de la zona de Arguineguín// que son preciosos ${ }^{27}$ porque los barrancos ahi son muy bonitos// (LPMla)

4) Comentario explicativo. La causal introduce un razonamiento que se distingue de los casos precedentes por su menor fuerza argumentativa ${ }^{28}$, pero que el hablante presenta como una reflexión válida para justificar la enunciación de algún segmento discursivo:

(25) la casa de Las: Canteras lo bueno es que estaba en primera línea de la playa/ que era una preciosidad subir a la azotea aquella// ver aquellas amanecidas y aquellas anochecidas y tal/ (LPH2b)

${ }^{26}$ Emitida con inflexión tonal descendente antes de porque.

27 Emitida por la informante con inflexión tonal después de preciosos.

${ }^{28}$ Desde nuestro punto de vista, las causales de la enunciación ofrecen, en la oralidad, una amplia casuística donde pueden percibirse diferentes grados de fuerza argumentativa, conformando una escala de mayor a menor en la que la hipótesis lógico-deductiva representa el máximo exponente (vid. Pérez Gil 2017: 285-286). 
5) Información conocida. Para justificar la enunciación se recupera una información supuestamente conocida por el interlocutor:

(26) ... íbamos a Teror (1") íbamos a ver la Virgen// y luego arrancábamos el musgo/ porque sabes que en Teror habia bero:les y mu:sgo// (LPM3b)

6) Contextualización. La explicación puede consistir, igualmente, en la inserción de un dato concreto que el hablante cree necesario dar a conocer para otorgar sentido -mediante su adecuada contextualización- a un acto enunciativo y guiar así al receptor en su interpretación:

(27) ... y la universidad muy bien m:uy bien// mi facultad de Medicina/ estaba estaba bastante bien pero// no estaba muy muy politizada ${ }^{29}$ porque en esa época// la política:/ influía muchísimo era// hacia el final del franquismo todavía (LPM3b)

7) Contraste u oposición. En estos casos, la estrategia argumentativa reside en el contraste que surge a partir de una comparación (en el ejemplo, ahora/antes):

(28) ... ahora hay cinco o seis catedráticos en: un mismo instituto y no se sabe quién/ quién va a ser el jefe:// de: de seminario o departamento/ porque antes lo lógico era el catedrático pues claro ha demostrado unos conocimientos era el jefe de departamento pues ahora// (e:) (LPM3a)

8) Metalingüísticas. La explicación se centra en la enunciación de un término o una expresión concretos ${ }^{30}$ :

(29) ... yo/ hay cosas que reconozco:// (m:) personalmente que (m:)// han conseguido este mi sucesora ${ }^{31}$ porque es una mujer $\mathrm{X} / /$ (LPH3a)

Puede suceder que la explicación no aluda a la elección de un término, sino al hecho de descartarlo (30), o incluso que tenga como fin justificar un silencio discursivo (31):

(30) ... dentro además a lo mejor se encuentra uno un bar// o mira hacia arriba y y sobre una:// ru- (m:) / (m:) ruinas no ${ }^{32}$ porque si no no podría ser ivverdad? pero an-/ (e:)// elementos de antigüedad/ (LPM3a)

(31) I: en cajitas de:/ de esas de madera de: de: (1") es que no me [acuerdo]

E: [de conserva]

I: de conserva (LPM3b)

${ }^{29}$ Aunque no hay pausa, las inflexiones tonales crean una frontera entonativa.

${ }^{30}$ De este tipo de causales han dado cuenta, entre otros, Galán (1999), Gutiérrez Ordóñez (2000) y la RAE-ASALE (2009).

${ }^{31}$ Si bien no hay pausa, sí existe frontera entonativa.

32 Porque va aquí precedido de inflexión tonal. 
En ocasiones, la causal metalingüística explica la enunciación de una expresión metafórica, como en el siguiente caso, en el que la informante describe un viaje por tierras asiáticas:

(32) ... es impresionante/ los edificios// el: aquello era/ era// [ah:]

E: [iqué bueno!]

I: ay// veías a Marco Polo por allí/

E: ¡qué bue:no!/ [iqué bue:no!]

I: [(risas) que es que] lo veías// porque/ era// en prin- muy [bonito]// (LPM3b)

9) Causales referidas a segmentos de modalidad no asertiva. Se trata, junto con la causa lógica o hipotética, del tipo de causal de la enunciación más frecuente en los ejemplos aportados por las gramáticas, si bien su consideración no resulta unánime ${ }^{33}$. Son algunos ejemplos hallados en nuestras muestras los siguientes:

(33) ... nosotros eso de las dudas en lugar de ahuyentar// el: los alumnos [...] les decíamos/ "vengan// porque mientras uno (golpes en la mesa) hace una pregunta de una duda ustedes están aprendiendo"// (LPM3a)

(34) E: [ah pues] cuéntame/ [y: y:]

I: [uf]// ¿qué te cuento de Samarcanda? es que todo lo que te diga es bonito/ (LPM3b)

La clasificación de todas las causales de la enunciación halladas en nuestro corpus nos proporciona los siguientes datos numéricos:

CuAdro 4. Modalidades de causales de la enunciación

\begin{tabular}{lrc}
\hline Tipo DE JUSTIFICACIÓN (215 casos) & $\mathrm{N}$ & $\%$ \\
\hline 1. Lógico-deductivas & 9 & 4,2 \\
2. Prueba o constatación & 43 & 20 \\
\hline 3. Paráfrasis & 8 & 3,7 \\
4. Comentario explicativo & 48 & 22,3 \\
5. Información conocida & 4 & 1,9 \\
6. Contextualización & 57 & 26,5 \\
7. Contraste u oposición & 12 & 5,6 \\
8. Metalingüísticas & 20 & 9,3 \\
9. Con segmentos no asertivos & 14 & 6,5 \\
\hline
\end{tabular}

${ }^{33}$ En este sentido, ya hemos advertido cómo Gutiérrez Ordóñez (2000) no considera las causales directivas causales de verbo enunciativo. 
Los dos porcentajes de uso más elevados corresponden, por tanto, a causales de la enunciación sustentadas, o bien en la transmisión de algún dato que permita al destinatario enmarcar la enunciación en el contexto adecuado para su interpretación $(26,5 \%)$, o bien en un comentario explicativo que pretende ser justificativo de la enunciación $(22,3 \%)$, mientras que los ejemplos que más abundan en los estudios teóricos (causales hipotéticas y con segmentos discursivos de modalidad no asertiva) tienen una representación mucho más reducida en nuestro corpus (de un 4,2\% y un $6,5 \%$, respectivamente $)^{34}$.

Un lugar común en la caracterización de todas las causales explicativas lo constituye la idea de que estas son la manifestación de un proceso argumentativo por el que el hablante se sirve de algún supuesto para presentar como adecuada o válida una explicación o justificación. La estrecha relación existente entre la causa y la argumentación está muy presente tanto en los trabajos de Santos Río $(1981,2001)$ como en las reflexiones que sobre la causalidad desarrolla Gutiérrez Ordóñez $(2000)^{35}$, y es admitida explícitamente en la NGLE al tratar las causales explicativas, cuando señala que «estas oraciones constituyen recursos argumentativos en los que se omite una premisa implícita o un supuesto implícito» (RAE-ASALE 2009: 3464).

Simultáneamente, quienes se han ocupado de estudiar el español coloquial y conversacional coinciden en considerar la argumentación un procedimiento subyacente en la mayoría de las interacciones verbales. Fuentes Rodríguez, por ejemplo, destaca cómo «no es exclusiva de un tipo de textos expositivos, de ensayo o de debate, sino que, por el contrario, está íntimamente ligada al habla» (1998: 119). Y Briz, que incluye la argumentación entre las estrategias conversacionales de las que se sirven los hablantes para alcanzar sus objetivos discursivos, la considera «el soporte en general del diálogo» (2001: 178). Este último autor, además, distingue cinco funciones argumentativas básicas, una de ellas consistente en la justificación, que hace residir en conectores pragmáticos como porque, es que, ya que, pues o puesto que -propios de las causales explicativas-. Todo ello indica que, debido a su carácter justificativo, las causales explicativas constituyen uno de los mecanismos de los que el hablante dispone para establecer las relaciones

${ }^{34}$ Sí presentan, no obstante, un porcentaje significativo (20\%) las causales de la enunciación basadas en la aportación de una prueba o constatación, que, aunque no coinciden plenamente con las lógico-deductivas, se hallan próximas a la causa lógica.

${ }^{35}$ Mientras que el primero de ellos deja constancia de «la íntima conexión de lo explicativo con la idea de argumentación» (2001: 446), Gutiérrez Ordóñez considera que «las oraciones causales son una manifestación o concreción lingüística de la función argumentativa del lenguaje» (2000: 103). 
argumentativas que dotan de coherencia a su discurso. Teniendo en cuenta, por tanto, que la argumentación es un procedimiento inherente al habla, no parece arriesgado apuntar una relación directa entre la capacidad argumentativa de las causales explicativas y su elevado índice de frecuencia. Dichas causales, a diferencia de las no explicativas, cumplen una función fundamental en el proceso conversacional, donde la justificación de lo que se dice (explicativas del enunciado) o del hecho de decirlo (explicativas de la enunciación) se convierte en una de las tácticas más recurrentes para orientar al interlocutor en la correcta interpretación de los enunciados y, en definitiva, de la intención comunicativa final.

Pero, en no pocas ocasiones, al valor argumentativo que conllevan estas construcciones se suma otra propiedad relacionada con los recursos comunicativos: la atenuación. Con la inserción de fórmulas atenuantes en su discurso, el hablante reduce la fuerza significativa de una expresión o la fuerza ilocutiva de un acto de habla y evita posibles tensiones (vid. Briz 2001: 143-163). En la bibliografía especializada, las causales explicativas aparecen a menudo vinculadas al deseo de mitigar lo dicho con una justificación, que puede ir introducida, entre otras, por partículas como porque, como o es que $e^{36}$. Entre los contextos que favorecen las explicativas con valor atenuante, encontramos, en nuestro corpus, ejemplos donde la justificación se añade tras una opinión sobre un tema conflictivo o que podría crear desacuerdo:

(35) ... lo que n:o me merece una// ta-/ tanta aprobación/ es la adopción por parte de los matrimonios homosexuales// porque: creo que:/ que bueno/ que:// el ni-/ un niño// tiene que tener el derecho a tener un padre y una madre// (LPM1b) ${ }^{37}$

El empleo de las causales explicativas como recursos de atenuación puede apreciarse, igualmente, en (12), donde se consigue suavizar la negativa dada a la encuestadora; en (14), en la que la atenuación contribuye a salvaguardar la imagen de la informante; en (33), donde la causal de la enunciación atenúa la fuerza ilocutiva de la forma de imperativo precedente

${ }^{36}$ En la «Ficha metodológica para el análisis pragmático de la atenuación en corpus discursivos del español» realizada en el marco del proyecto ES.POR.ATENUACIÓN se alude directamente a las causales de la enunciación entre las «construcciones justificadoras o de excusa» (Albelda et al. 2014). El uso de construcciones causales como mecanismos de atenuación queda reflejado también en trabajos como los de Briz (2001) o Albelda y Briz (2010).

${ }^{37}$ Puede observarse cómo la justificación se combina con otros elementos de atenuación, como la elección de la expresión «lo que no me merece tanta aprobación» en lugar de otra más directa («Soy contraria a», «No estoy de acuerdo con», etc.) o la forma verbal «creo» de la causal. 
(«vengan») orientando su interpretación hacia el consejo o la recomendación; o, incluso, en enunciados suspendidos como el de $(21)^{38}$. Los casos extraídos de nuestras muestras indican, por tanto, la conveniencia de atender a su posible papel atenuante al estudiar los factores que pueden incidir en el predominio de las causales explicativas frente a las restantes construcciones causales en el habla ${ }^{39}$.

Finalmente, la configuración de la muestra analizada nos permite organizar los datos en relación con el sexo y la edad. En lo que se refiere a la primera de esas variables, obtuvimos los datos recogidos a continuación:

CuAdro 5. Causales no explicativas y explicativas según el factor sexo

\begin{tabular}{l|cc|cc}
\hline \multirow{2}{*}{ ENUNCIADOS CAUSALES } & \multicolumn{2}{|c|}{ Mujeres } & \multicolumn{2}{c}{ Hombres } \\
\cline { 2 - 5 } & $\mathrm{N}$ & $\%$ & $\mathrm{~N}$ & $\%$ \\
\hline Causales no explicativas & 65 & 13,5 & 64 & 15,9 \\
\hline Causales explicativas & 418 & 86,5 & 338 & 84,1 \\
\hline TotaL & 483 & & 402 \\
\hline
\end{tabular}

Las cifras reflejadas destacan por su extraordinaria simetría: por un lado, las causales explicativas constituyen el grupo mayoritario tanto en las mujeres como en los hombres; por otro, su representación porcentual es muy similar. Si bien el porcentaje más alto lo ofrece el habla femenina $(86,5 \%)$, la ventaja cuantitativa con respecto a los hablantes masculinos no puede considerarse relevante, pues alcanza únicamente 2,4 puntos porcentuales $^{40}$.

En cuanto al factor edad, la distribución que presentan las tres generaciones contempladas es la siguiente:

${ }^{38}$ En relación con el valor atenuante de las causales suspendidas con como, vid. Montolío (2001).

${ }^{39}$ A este respecto, resulta significativo que, en un trabajo sobre el análisis de las fórmulas de atenuación usadas en el español de Las Palmas de Gran Canaria, la introducción de justificaciones y excusas ocupe el segundo lugar de frecuencia de aparición (vid. Samper Hernández 2013).

${ }^{40}$ Estos datos no corroboran, por tanto, el mayor carácter cortés y cooperativo que tradicionalmente se ha atribuido al habla de las mujeres (vid. Lozano 1995, García Mouton 1999 o Acuña 2011). 
CuAdro 6. Causales no explicativas y explicativas según el factor edad

\begin{tabular}{|c|c|c|c|c|c|c|}
\hline \multirow[b]{2}{*}{ ENUNCIADOS CAUSALES } & \multicolumn{2}{|c|}{ 1. a generación } & \multicolumn{2}{|c|}{ 2. a generación } & \multicolumn{2}{|c|}{ 3. a generación } \\
\hline & $\mathrm{N}$ & $\%$ & $\mathrm{~N}$ & $\%$ & $\mathrm{~N}$ & $\%$ \\
\hline Causales no explicativas & 33 & 17,4 & 53 & 16,2 & 43 & 11,7 \\
\hline Causales explicativas & 157 & 82,6 & 274 & 83,8 & 325 & 88,3 \\
\hline TOTAL & 190 & & 327 & & 368 & \\
\hline
\end{tabular}

En todos los grupos etarios, las causales explicativas presentan un porcentaje de uso superior al $80 \%$. Aunque el índice más elevado (88,3\%) corresponde a los hablantes de la tercera generación, que son, a su vez, quienes aportan el mayor número de casos, solo 5,7 puntos porcentuales separan los dos extremos (primera y tercera generación), por lo que tampoco esta variable se muestra determinante en la elección de unas u otras causales.

\section{Conclusiones}

Las cifras resultantes de nuestro corpus reflejan, en primer lugar, una importante diferencia cuantitativa en relación con las distintas clases de causales, que sitúa claramente a las explicativas como el grupo causal preferente en la conversación. Mientras que las causales no explicativas representan únicamente el 14,6\% del total, las causales del enunciado explicativas sobrepasan la mitad del conjunto $(61,1 \%)$; a estas últimas ha de sumarse, además, el 24,3\% correspondiente a las causales de la enunciación, donde el rasgo explicativo es una constante. Por tanto, en el 85,4\% de los casos analizados, el hablante se sirve del segmento causal para transmitir, además, algún tipo de explicación, que en la mayoría de las ocasiones $(71,6 \%)$ justifica lo dicho (frente al $28,4 \%$ de explicativas de la enunciación).

El análisis pormenorizado de los enunciados causales explicativos de nuestro corpus (756 casos) nos ha proporcionado una información muy relevante a la hora de determinar los contextos a los que, normalmente, se vinculan esas construcciones en la oralidad. Atendiendo al objetivo de la justificación expresada, hemos establecido hasta 10 posibles factores contextuales, si bien la mayoría de los casos se distribuyen entre la explicación de una información que el informante considera adecuado aclarar (ya sea por la sorpresa que pudiera causar en el interlocutor, por poner en riesgo 
la imagen del hablante, por su complejidad, etc.), que dio lugar al 38,1\% de los enunciados causales explicativos, y la necesidad de justificar el propio acto enunciativo (finalidad a la que responde el 28,4\%). En lo que se refiere a este último subgrupo, coincidente con las tradicionales «causales de la enunciación», la explicación consiste, en la mayoría de las ocasiones, en aportar un dato, a priori desconocido por el interlocutor, orientativo de la contextualización que otorga sentido a la enunciación $(26,5 \%)$ o en hacer explícita una reflexión que, sin alcanzar la categoría de presupuesto lógico o deductivo, se presenta como válida para justificar el acto enunciativo $(22,3 \%)$, en contraste con los ejemplos característicos para esta clase de causales de las gramáticas (causales lógicas y justificaciones referidas a fragmentos discursivos con una modalidad diferente a la asertiva), que tienen una representación minoritaria en las muestras de habla analizadas.

Pero el estudio de una construcción sintáctica -cualquiera que esta seaen la oralidad no debe desarrollarse al margen, hoy, de los importantes avances alcanzados por los especialistas en el análisis del discurso oral, que han puesto de manifiesto la existencia de diversas estrategias subyacentes a toda actividad conversacional. La naturaleza justificativa de las causales explicativas aconseja contemplar, en este sentido, su estrecha relación con dos estrategias conversacionales fundamentales en la interacción lingüística: la argumentación y la atenuación. El carácter argumentativo inherente a toda causal explicativa, por un lado, y su posible papel como mecanismo de atenuación en ciertos contextos, por otro, se presentan como dos factores lingüísticos significativos a la hora de analizar el elevado índice de frecuencia de estos enunciados en el habla y ofrecen, creemos, una línea de investigación interesante para continuar profundizando en la caracterización de esos enunciados. Por el contrario, las variables sociales analizadas en nuestro trabajo no se mostraron influyentes, ya que ni el sexo ni la edad determinaron diferencias relevantes en cuanto al uso de uno u otro tipo de causales. 


\section{BIBLIOGRAFÍA}

ACUÑa FerReIRA, A. Virginia (2011): La realización de quejas en la conversación femenina y masculina. Diferencias y semejanzas en el habla cotidiana de las mujeres y los hombres, Múnich: Lincom Europa.

Albelda Marco, Marta y Antonio Briz Gómez (2010): «Aspectos pragmáticos. Cortesía y atenuantes verbales en las dos orillas a través de muestras orales». En Milagros Aleza Izquierdo y José M. ${ }^{a}$ Enguita Utrilla (coords.), La lengua española en América: normas y usos actuales, Valencia: Universidad de Valencia, 237-260.

—, - Ana M. ${ }^{a}$ Cestero, Dorota Kotwica y Cristina Villalba (2014): «Ficha metodológica para el análisis pragmático de la atenuación en corpus discursivos del español (ES.POR.ATENUACIÓN)», Oralia 17, 7-62.

Bello, Andrés ([1847] 1981): Gramática de la lengua castellana (ed. crítica a cargo de R. Trujillo), Santa Cruz de Tenerife: Cabildo Insular de Tenerife.

BRIz Gómez, Antonio (2001): El español coloquial en la conversación. Esbozo de pragmagramática, 2. ${ }^{\mathrm{a}}$ ed., Barcelona: Ariel.

Caravedo, Rocío (2003): «Causalidad en el discurso oral». En José Luis Girón Alconchel et al. (eds.), Estudios ofrecidos al profesor José Jesús de Bustos Tovar, Madrid: Editorial Complutense, I, 45-60.

Carbonero Cano, Pedro (2001): «Cuestiones de sintaxis oral: causales, explicativas y justificativas». En Elena Méndez García de Paredes et al. (eds.), Indagaciones sobre la lengua. Estudios de filología y lingüistica españolas en memoria de Emilio Alarcos, Sevilla: Universidad de Sevilla, 313-322.

Fuentes Rodríguez, Catalina (1998): «Acercamiento a ciertos procesos argumentativos del texto oral», Oralia 1, 119-139.

GALÁN RodRíGUEZ, Carmen (1995): «Las oraciones causales: propuesta de clasificación», Anuario de Estudios Filológicos 18, 125-158.

- (1999): «La subordinación causal y final». En Ignacio Bosque Muñoz y Violeta Demonte Barreto (dirs.), Gramática descriptiva de la lengua española, Madrid: Espasa, 3597-3642.

García García, Serafina (1996): Las expresiones causales y finales, Madrid: Arco/ Libros.

García Mouton, Pilar (1999): Cómo hablan las mujeres, Madrid: Arco/Libros.

Grande Alja, Francisco Javier y M. ${ }^{a}$ del Carmen Lanero Rodríguez (2019): «La modalidad de enunciación en las causales explicativas: una aproximación macrosintáctica». En Catalina Fuentes Rodríguez y Salvador Gutiérrez Ordóñez (eds.), Avances en macrosintaxis, Madrid: Arco/Libros, 225-256.

GILI GaYA, Samuel ([1943] 1990): Curso superior de sintaxis española, Barcelona: Vox.

Gutiérrez Ordóñez, Salvador (2000): «Causales», Boletín de la Real Academia Española $80 / 279,47-159$. 
- (2002): «Comentario sintáctico de un texto de Pío Baroja». En Salvador Gutiérrez Ordóñez, Forma y sentido en sintaxis, Madrid: Arco/Libros, 209-247.

Hernández Cabrera, Clara Eugenia y José Antonio SAmper Padilla (2012): «La investigación sociolingüística en el marco del PRESEEA en la ciudad de Las Palmas de Gran Canaria». En José Manuel Marrero Henríquez et al. (eds.), La luz no interrumpida. Homenaje a Eugenio Padorno, Madrid: Ediciones Clásicas, 393-404.

IGLESIAS BANGO, Manuel (1997): «La oposición enunciado/enunciación y las llamadas subordinadas adverbiales impropias en español», Moenia 3, 237-269.

LAPESA, Rafael (1978): «Sobre dos tipos de subordinación causal». En Estudios ofrecidos a Emilio Alarcos Llorach, Oviedo: Universidad de Oviedo, III, 173-205.

LÓPEz GARCíA, Ángel (1994): Gramática del español. I. La oración compuesta, Madrid: Arco/Libros.

LozAno Domingo, Irene (1995): Lenguaje femenino, lenguaje masculino. ¿Condiciona nuestro sexo la forma de hablar?, Madrid: Minerva ediciones.

Montolío Durán, Estrella (2001): «Sobre el valor discursivo de la construcción causal [como A, B]». En José Jesús de Bustos Tovar et al. (eds.), Lengua, discurso, texto (I Simposio Internacional de Análisis del Discurso), Madrid: Visor Libros, 359-381.

NARBONA JimÉNEZ, Antonio (1990): Las subordinadas adverbiales impropias en español (II) (Causales y finales, comparativas y consecutivas, condicionales y concesivas), Málaga: Ágora.

PÉrez GIL, Otilia (2017): Las causales en la oralidad. Los enunciados con «porque», Salamanca: Ediciones Universidad Salamanca.

RAE (1973): Esbozo de una nueva gramática de la lengua española, Madrid: EspasaCalpe.

RAE-ASALE (2005): Diccionario panhispánico de dudas (DPD), Madrid: Santillana.

- (2009): Nueva gramática de la lengua española (NGLE), Madrid: Espasa Libros.

SAMPER HERNÁNDEZ, Marta (2013): «La atenuación lingüística en el español de Las Palmas de Gran Canaria», Lingüistica Española Actual 35/2, 325-348.

SÁNCHEZ MÁrQuez, Manuel J. (1972): Gramática moderna del español. Teoría y norma, Buenos Aires: Ediar.

SANTOS Río, Luis (1981): "Reflexiones sobre la expresión de la causa en castellano», Studia Philologica Salmanticensia 6, 231-277.

- (1993): «Explicatividad: algunas puntualizaciones sobre los nexos y las proposiciones que la expresan». En Indagaciones semánticas, sintácticas y lexicográficas, Salamanca: Gráficas Varona, 31-36.

- (2001): «Causa explicativa y estructuración del discurso». En José Jesús de Bustos Tovar et al. (eds.), Lengua, discurso, texto (I Simposio Internacional de Análisis del Discurso), Madrid: Visor Libros, 445-469. 